\title{
Coronary Bypass Grafting in Acute Coronary Syndrome: Operative Approaches and Secondary Prevention
}

\author{
Stephen J. Huddleston ${ }^{1}$ and Gregory Trachiotis ${ }^{2}$ \\ 1The Johns Hopkins Hospital, \\ ${ }^{2}$ George Washington University and Veterans Affairs Medical Center \\ United States of America
}

\section{Introduction}

Many patients who present with acute coronary syndrome (ACS) are candidates for coronary artery bypass grafting (CABG). Approximately 500,000 CABG operations are performed each year in the United States, and many of these patients present with acute coronary syndrome. In the Bypass Angioplasty Revascularization Investigation (BARI) trial comparing CABG and percutaneous coronary intervention for coronary artery disease, $64 \%$ of the patients presented with unstable angina (Rosamond, Flegal et al. 2008). According to 2007 ACC/AHA guidelines (Anderson, Adams et al. 2007), left main stenosis greater than $50 \%$ and three-vessel coronary artery disease represent a class I indication for coronary artery bypass grafting in patients with unstable angina or non-ST elevation myocardial infarction. In addition, patients with 2-vessel coronary artery disease and either diabetes or left ventricular dysfunction may benefit from coronary artery bypass grafting (Class II recommendation). Regardless of the modality of revascularization, patients with acute coronary syndrome benefit from antagonists of platelet activity such as aspirin and clopidogrel since these agents reduce the risk of major adverse events. However, antiplatelet agents also increase the risk of bleeding in patients who will ultimately proceed to coronary artery bypass graft surgery. Usually the benefits of early initiation of antiplatelet therapy outweigh the risks. The purpose of this review is to summarize current antiplatelet therapy in acute coronary syndrome, with particular emphasis on patients who will undergo coronary artery bypass, and the appropriate operative strategies will be discussed. We will also review the current recommendations for secondary prevention after coronary artery bypass grafting.

\section{Antiplatelet therapy}

Platelet activation and aggregation play a critical role in the pathogenesis of coronary thrombosis, and antiplatelet therapy has become a central tenet of the treatment strategy in acute coronary syndrome. Platelet aggregation and activation can be inhibited pharmacologically at several points in the cascade leading up to platelet aggregation, and several of these antiplatelet agents are commonly used perioperatively in the setting of acute 
coronary syndrome. Aspirin inhibits the formation of thromboxane A2 thereby preventing glycoprotein (Gp) IIb/IIIa-mediated activation of platelets. Ticlodipine and clopidogrel inhibit ADP release from platelets and thereby prevent $\mathrm{Gp} \mathrm{IIb/IIIa} \mathrm{activation,} \mathrm{and}$ eptifibatide blocks GpII B/IIIa-mediated platelet aggregation. The pharmacokinetics of platelet inhibition and inactivation by each type of agent is of particular importance regarding the timing of $C A B G$ because of the bleeding risk associated with antiplatelet therapy. However, the timing of CABG in the setting of ACS is often flexible, with perhaps less than $1 \%$ of patients with ACS requiring emergent CABG (Serebruany, Malinin et al. 2004), so much of the bleeding risk associated with antiplatelet therapy can often be mitigated by waiting several days before CABG is performed.

Regardless of whether patients with ACS undergo percutaneous coronary intervention or CABG, the overwhelming body of evidence supports the use of antiplatelet therapy in ACS. In one large meta-analysis of studies of patients with ACS (Seshadri, Whitlow et al. 2002), the risk of serious vascular events including non-fatal myocardial infarction or stroke was reduced by $25 \%$. Although aspirin was associated with a significantly reduced risk of major vascular events, the adenosine diphosphate (ADP)-receptor antagonists ticlodipine and clopidogrel were associated with an additional $10-12 \%$ risk reduction. In a direct comparison of aspirin and clopidogrel in the randomized Clopidogrel versus Aspirin in Patients at Risk for Ischemic Events (CAPRIE) trial, clopidogrel was associated with an $8.7 \%$ relative reduction of risk of ischemic stroke, myocardial infarction, or vascular death compared with aspirin, with no signicant difference in the safety profiles of the two drugs (1996). Furthermore, the beneficial effects of aspirin and clopidogrel in ACS are additive. In the Clopidogrel in Unstable angina to prevent Recurrent Events (CURE) trial, unstable angina and non-ST elevation myocardial infarction (NSTEMI), patients taking both clopidogrel and aspirin had less chance of cardiac death, non-fatal MI, or stroke at 30 days and 1 year compared to those only taking aspirin (Yusuf, Zhao et al. 2001). Taken together, these studies all confirm that antiplatelet therapy with both aspirin and clopidogrel should be intitiated early in ACS.

In patients with ST-elevation myocardial infarction (STEMI), clopidogrel in addition to aspirin improves outcomes. In the Clopidogrel and Metoprolol in Myocardial Infarction (COMMIT) trial, clopidogrel in addition to aspirin was associated with a significant decrease in the composite endpoint of death, reinfarction, or stroke compared to aspirin alone (Sabatine, Cannon et al. 2005). The Clopidogrel as Adjunctive Reperfusion TherapyThrombolysis In Myocardial Infarction (CLARITY-TIMI) compared aspirin alone to clopidogrel in addition to aspirin in patients with STEMI and demonstrated a 36\% reduction in relative risk of the early composite endpoint of occlusion of the infarct-related artery, recurrent MI, or death, and there was a $20 \%$ relative risk reduction of the late composite endpoint of death from cardiovascular cause, recurrent MI, or recurrent ischemia leading to urgent revascularization (Sabatine, Cannon et al. 2005). Importantly, there was no increase in bleeding complications in either the COMMIT or the CLARITY-TIMI trial. Based on such compelling findings, the current AHA/ACC guidelines for patients with ACS recommend that aspirin and clopidogrel be given as soon as possible (Anderson, Adams et al. 2007). The typical loading dose for clopidogrel is $300 \mathrm{mg}$ or $600 \mathrm{mg}$. Usually for patients undergoing stent placement, $600 \mathrm{mg}$ of clopidogrel is given, while patients who are found to be candidates for CABG are given $300 \mathrm{mg}$ of clopidogrel.

New ADP-receptor antagonist antiplatelet agents include prasugrel and ticagrelor. Both decrease the relative risk of ischemic events when used with aspirin (Ebrahimi, Dyke et al. 
2009; Wallentin, Becker et al. 2009). However, prasugrel is associated with increased risk of bleeding complications, especially related to CABG. In contrast, ticagrelor was not associated with increased risk of bleeding related to CABG.

Several clinical trials have examined the outcomes of patients with ACS who were treated with antiplatelet therapy before undergoing CABG. In the CURE trial, patients who proceeded to $\mathrm{CABG}$ after receiving clopidogrel as opposed to placebo had a decreased relative risk of the composite endpoint of death, MI or stroke prior to CABG (RR $0.5695 \%$ CI $0.29-1.08$, absolute risk reduction 1.8\%). As long as clopidogrel was stopped 5 days prior to CABG there was no increase in risk of life-threatening bleeding. The CLARITY-TIMI trial and the ACUITY (Acute Catheterization and Urgent Intervention Triage strategy) trials demonstrated similar improved outcomes without increased risk of bleeding in patients who underwent CABG after being treated with clopidogrel (Ferraris, Ferraris et al. 2005).

\section{Coronary artery bypass grafting and acute coronary syndrome}

Using antiplatelet agents in patients with ACS raises the risk of bleeding in those patients that undergo CABG, however several factors including the pharmacokinetic profile, individual patient sensitivity to the agent, and timing of the surgery determine the exact risk posed. Clinical trials are often difficult to compare because of varying classification of bleeding complications leading to differing conclusions. The Aspirin Guideline Taskforce of the Society of Thoracic Surgeons reviewed 21 studies examining the effect of preoperative aspirin on bleeding after CABG (Ebrahimi, Dyke et al. 2009). The dose of aspirin varied significantly between studies, so comparison of the findings is difficult. However, there appears to be a small increase in chest tube drainage and blood loss and a small increase in need for blood transfusion associated with preoperative aspirin, all of which may be dose related. Nonetheless, the mortality benefit of aspirin preoperatively in ACS outweighs the small risk of bleeding, leading to a class IIa recommendation to continue aspirin for urgent/emergent CABG patients. Some studies have shown an increased risk of perioperative bleeding in patients treated with both aspirin and ADP receptor antagonists such as clopidogrel who proceed to CABG (Yende and Wunderink 2001; Hongo, Ley et al. 2002; Genoni, Tavakoli et al. 2003). Therefore, clopidogrel should be stopped for 5-7 days before CABG when possible (ACC/AHA class I recommendation).

Because of the increased risk of bleeding after CABG in patients on dual antiplatelet therapy, one strategy would be to wait until coronary anatomy is defined on coronary angiography. However, this would delay antiplatelet therapy for all patients with a decrement in the benefit for all patients, most of whom do not end up undergoing CABG. The number of patients with ACS who proceed to CABG is probably between 7-14\% (Bhatt, Roe et al. 2004; Fox, Anderson et al. 2007), and salvage CABG after failed percutaneous coronary intervention is relatively uncommon. The risk of bleeding after CABG, as well as the risk of reoperation for bleeding, overall is fairly low. Thus, the vast majority of patients will benefit from starting dual antiplatelet therapy early in ACS, and many patients would be harmed by delaying therapy.

\section{Operative strategies}

Usually CABG can be delayed in ACS to allow ADP-receptor blockers to be metabolized and excreted allowing platelet function to recover. If anticoagulation is critical, as is usually 
the case in ACS, short acting anticoagulants such as unfractionated heparin and Gp IIb/IIIa inhibitors can be used up until shortly before going to the operative room. Patients with ACS who have ongoing ischemia or are hemodynamically unstable and have received ADPreceptor antagonists may benefit from bridge percutaneous coronary intervention or intraaortic balloon pump insertion. An algorithm for the management of patients with ACS that undergo CABG is presented in Figure 1.

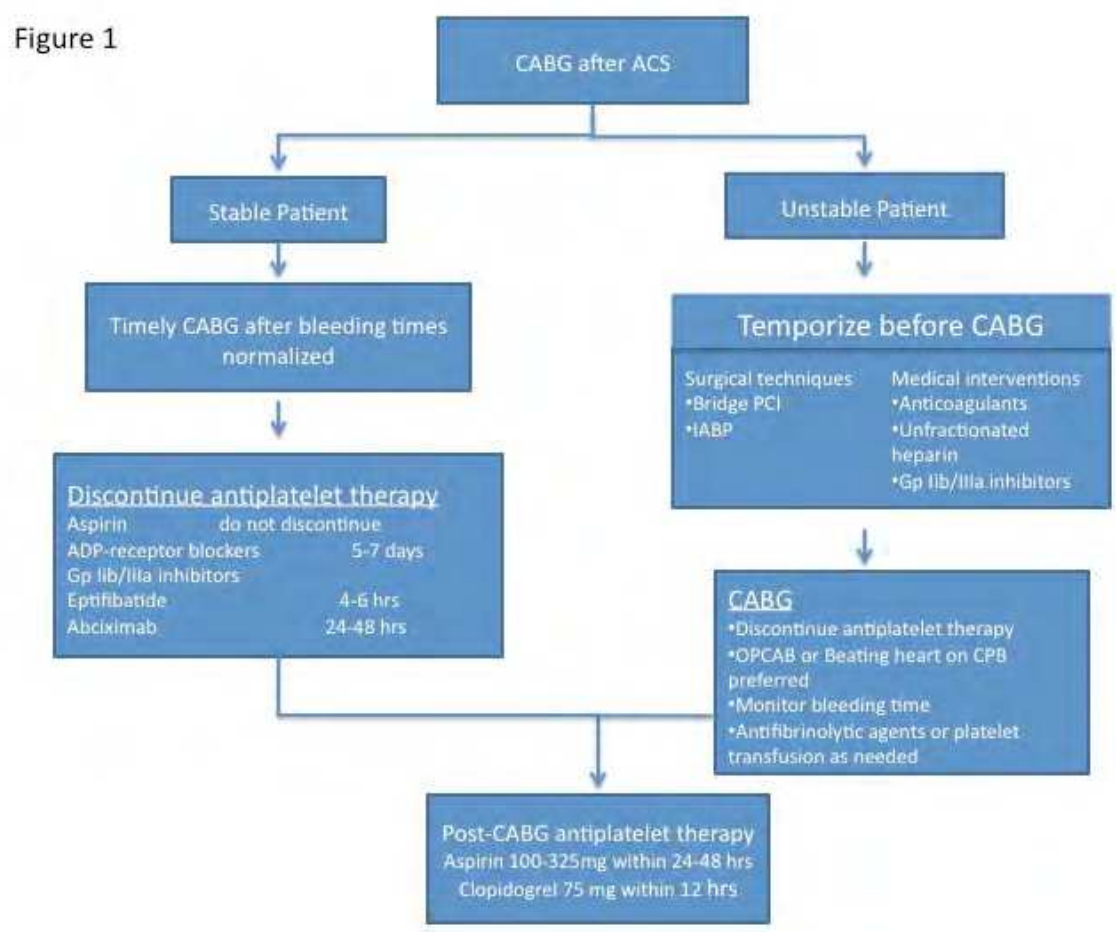

Fig. 1. Algorithm or the management of patients with ACS and indications for CABG.

Patients with ACS who are stable are observed while ADP receptor blockers are held for 5 days prior to surgery to eliminate the increased risk of bleeding and mortality (Ferraris, Ferraris et al. 2005; Ebrahimi, Dyke et al. 2009). Aspirin is continued up to the time of surgery. If there is particular concern about the grade of coronary stenosis or evidence of ongoing ischemia, the patient may be continued on unfractionated heparin and/or Gp $\mathrm{IIb} / \mathrm{III}$ inhibitors up until the time of surgery. The bleeding time or platelet function time can be used to assess platelet function and the bleeding risk preoperatively. This may be useful in assessing bleeding risk and planning for CABG. The dose of clopidogrel may be proportional to the risk of bleeding after CABG. In patients who receive the $600 \mathrm{mg}$ loading dose of clopidogrel, $\mathrm{CABG}$ should be delayed 5-7 days for the platelet function to recover. However, in patients who received the lower $300 \mathrm{mg}$ loading dose and are maintained on 75 mg per day of clopidogrel, CABG may be safely performed after holding clopidogrel for 1-3 days. A logical strategy is to administer $300 \mathrm{mg}$ of clopidogrel in addition to aspirin at the 
time of presentation in patients with ACS. When coronary angiography is performed, an additional $300 \mathrm{mg}$ of clopidogrel may be given if percutaneous coronary intervention is performed, while in those that will undergo CABG, no additional clopidogrel is given and low dose aspirin is continued.

In patients with ACS and high grade left main coronary artery stenosis or left main equivalent who have received high dose clopidogrel or thrombolytics, insertion of an intraaortic balloon pump (IABP) will improve coronary perfusion and decrease cardiac demand by afterload reduction. Preoperative IABP insertion in these situations provides hemodynamic stability and reduces hospital mortality (Wiviott, Braunwald et al. 2007).

CABG may be performed on cardiopulmonary bypass with cardioplegic arrest or off-pump $(\mathrm{OPCAB})$. The advantages and disadvantages of OPCAB have been studied and reviewed extensively, yet remain controversial (Moore, Pfister et al. 2005). OPCAB avoids full heparinization and the systemic inflammatory response syndrome associated with cardiopulmonary bypass. Early studies suggested that off-pump CABG is associated with fewer adverse cardiovascular events and reduced early postoperative morbidity and mortality (Rastan, Eckenstein et al. 2006; Magee, Alexander et al. 2008). However, more recent data demonstrate a slightly higher rate of incomplete revascularization and a greater need for subsequent revascularization, as well as slightly reduced long-term mortality (Puskas, Kilgo et al. 2008). However, much of the outcome after OPCAB may be surgeon and center dependent, and this should be taken into account when formulating operative plans for patients with ACS undergoing CABG.

\section{Secondary prevention}

CABG for patients with ACS reduces angina, reduces the risk of myocardial infarction, and improves long-term survival, particularly when the internal thoracic artery is grafted to the left anterior descending artery (Anderson, Adams et al. 2007). However, secondary prevention strategies are critical for the long-term benefit of revascularization to be realized. Lifestyle modification such as diet modification, an exercise regimen, and smoking cessation are necessary. Pharmacologic secondary prevention consisting of lipidlowering agents, antihypertensives (primarily with beta blockers), and antiplatelet medications is also required to decrease the risk of progression of coronary artery disease and graft occlusion. A strategy for antiplatelet therapy after CABG in patients with ACS is presented in Figure 2. All patients should be continued on aspirin postoperatively to reduce the risk of vein graft occlusion (ACC/AHA class I recommendation). This also protects against the risk of future cardiovascular, cerebrovascular, and peripheral vascular ischemic events. In patients with ACS who have undergone coronary artery stent placement, clopidogrel is required in addition to aspirin to reduce major cardiac events (Muller, Buttner et al. 2000). In the CURE trial, patients with ACS treated with clopidogrel in addition to aspirin had decreased cardiac events, stroke and hospitalizations at 1 year regardless of whether they underwent PCI or CABG (Yusuf, Zhao et al. 2001). Therefore, in our practice, patients with ACS who undergo on-pump CABG or OPCAB are treated with $600 \mathrm{mg}$ aspirin suppository upon postoperative admission to the intensive care unit. When they are extubated, clopidogrel $75 \mathrm{mg}$ daily is administered, in addition to aspirin $81 \mathrm{mg}$ daily. Dual antiplatelet therapy is continued in all patients with ACS for 9-12 months. 


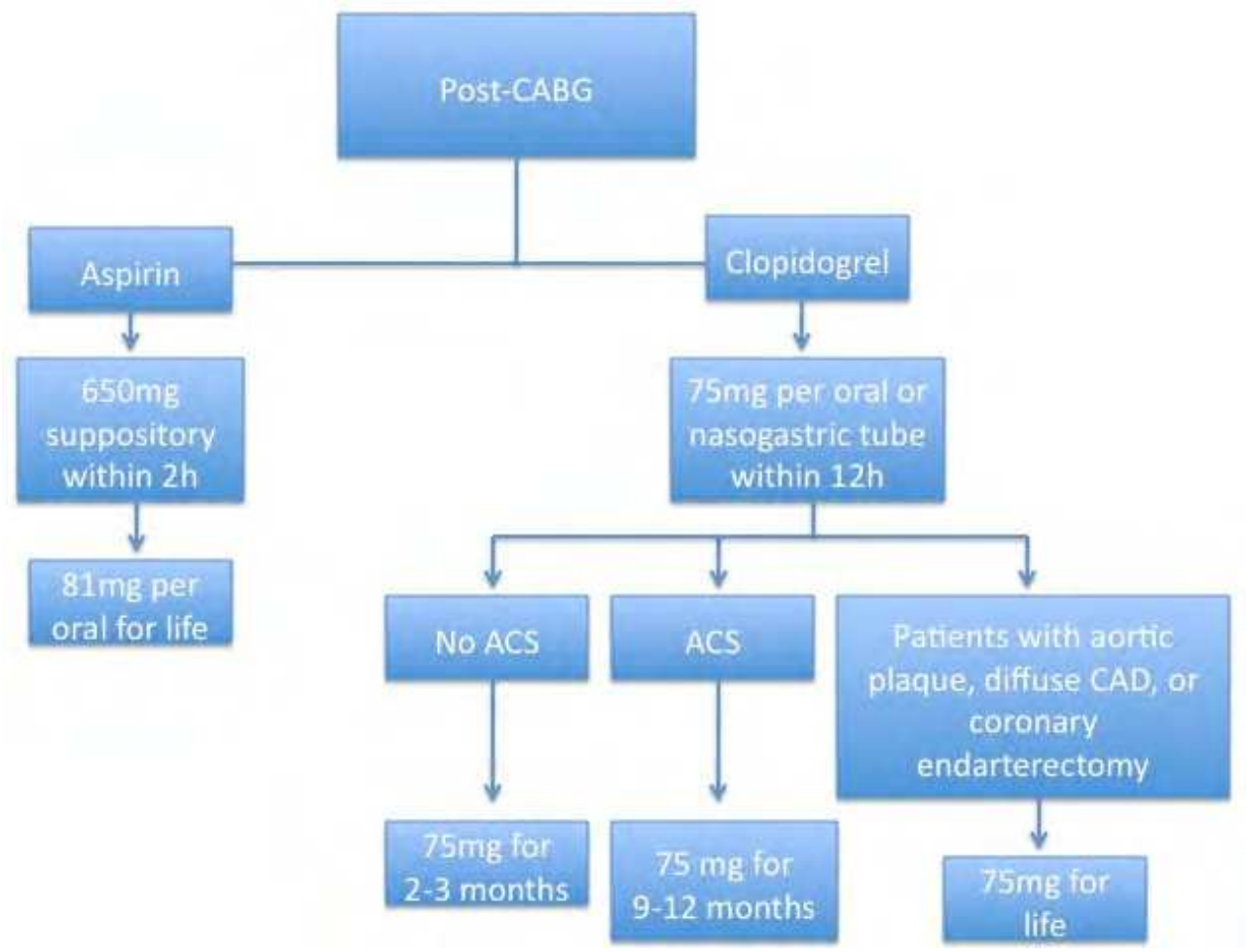

Fig. 2. Strategy for antiplatelet therapy for secondary prevention of CAD after CABG.

All patients with a history of a cardiovascular event should be started on a beta blocker and 3-hydroxy-3-methylglutaryl coenzyme A (MHG -CoA) reductase inhibitors (statins) for secondary prevention of cardiovascular events unless otherwise contraindicated (1994; Sacks, Pfeffer et al. 1996; 1998). Angiotensin converting enzyme inhibitors should be initiated in all patients with CAD and a left ventrticular ejection fraction less than $40 \%$, diabetes, hypertension, or chronic kidney disease (Smith, Allen et al. 2006). ACE inhibitors may be considered in lower risk patients with well-controlled risk factors. If ACE inhibitors are not tolerated or are contraindicated, angiotensin receptor blockers (ARBs) may be considered. Patients with heart failure may also benefit from ARBs. Medication compliance is required for successful secondary prevention and must be ensured to reap the benefits of revascularization.

\section{Conclusions}

Antiplatelet therapy with aspirin and clopidogrel imparts a clear benefit in major adverse cardiovascular events and mortality after acute coronary syndrome. Although these agents may pose additional bleeding risk in the small percentage of patients with ACS that proceed to $C A B G$, the risk is far outweighed by the benefits, and much of the bleeding can be diminished by delaying CABG when possible. Short acting anticoagulants and IABP insertion may temporize while allowing platelet function to recover after discontinuing 
clopidogrel. Following bleeding times postoperatively can clarify the need for platelet transfusion in the event of bleeding. OPCAB may have offer some benefits by avoiding full heparinization and avoiding the inflammatory response associated with cardiopulmonary bypass. Secondary prevention with antiplatelet therapy, beta blockade, lipid lowering therapy, and ACE inhibitors or ARBs is critical to the long term success of revascularization.

\section{References}

(1994). "Randomised trial of cholesterol lowering in 4444 patients with coronary heart disease: the Scandinavian Simvastatin Survival Study (4S)." Lancet 344(8934): 13839.

(1996). "A randomised, blinded, trial of clopidogrel versus aspirin in patients at risk of ischaemic events (CAPRIE). CAPRIE Steering Committee." Lancet 348(9038): 132939.

(1998). "Prevention of cardiovascular events and death with pravastatin in patients with coronary heart disease and a broad range of initial cholesterol levels. The LongTerm Intervention with Pravastatin in Ischaemic Disease (LIPID) Study Group." $N$ Engl J Med 339(19): 1349-57.

Anderson, J. L., C. D. Adams, et al. (2007). "ACC/AHA 2007 guidelines for the management of patients with unstable angina/non-ST-Elevation myocardial infarction: a report of the American College of Cardiology/American Heart Association Task Force on Practice Guidelines (Writing Committee to Revise the 2002 Guidelines for the Management of Patients With Unstable Angina/Non-ST-Elevation Myocardial Infarction) developed in collaboration with the American College of Emergency Physicians, the Society for Cardiovascular Angiography and Interventions, and the Society of Thoracic Surgeons endorsed by the American Association of Cardiovascular and Pulmonary Rehabilitation and the Society for Academic Emergency Medicine." J Am Coll Cardiol 50(7): e1-e157.

Bhatt, D. L., M. T. Roe, et al. (2004). "Utilization of early invasive management strategies for high-risk patients with non-ST-segment elevation acute coronary syndromes: results from the CRUSADE Quality Improvement Initiative." Jama 292(17): 2096104.

Ebrahimi, R., C. Dyke, et al. (2009). "Outcomes following pre-operative clopidogrel administration in patients with acute coronary syndromes undergoing coronary artery bypass surgery: the ACUITY (Acute Catheterization and Urgent Intervention Triage strategY) trial." J Am Coll Cardiol 53(21): 1965-72.

Ferraris, V. A., S. P. Ferraris, et al. (2005). "The Society of Thoracic Surgeons practice guideline series: aspirin and other antiplatelet agents during operative coronary revascularization (executive summary)." Ann Thorac Surg 79(4): 1454-61.

Fox, K. A., F. A. Anderson, Jr., et al. (2007). "Intervention in acute coronary syndromes: do patients undergo intervention on the basis of their risk characteristics? The Global Registry of Acute Coronary Events (GRACE)." Heart 93(2): 177-82.

Genoni, M., R. Tavakoli, et al. (2003). "Clopidogrel before urgent coronary artery bypass graft." J Thorac Cardiovasc Surg 126(1): 288-9.

Hongo, R. H., J. Ley, et al. (2002). "The effect of clopidogrel in combination with aspirin when given before coronary artery bypass grafting." J Am Coll Cardiol 40(2): 231-7. 
Magee, M. J., J. H. Alexander, et al. (2008). "Coronary artery bypass graft failure after onpump and off-pump coronary artery bypass: findings from PREVENT IV." Ann Thorac Surg 85(2): 494-9; discussion 499-500.

Moore, G. J., A. Pfister, et al. (2005). "Outcomes for off-pump coronary artery bypass grafting in high-risk groups: a historical perspective." Heart Surg Forum 8(1): E19-22.

Muller, C., H. J. Buttner, et al. (2000). "A randomized comparison of clopidogrel and aspirin versus ticlopidine and aspirin after the placement of coronary-artery stents." Circulation 101(6): 590-3.

Puskas, J. D., P. D. Kilgo, et al. (2008). "Off-pump coronary bypass provides reduced mortality and morbidity and equivalent 10-year survival." Ann Thorac Surg 86(4): 1139-46; discussion 1146.

Rastan, A. J., J. I. Eckenstein, et al. (2006). "Emergency coronary artery bypass graft surgery for acute coronary syndrome: beating heart versus conventional cardioplegic cardiac arrest strategies." Circulation 114(1 Suppl): I477-85.

Rosamond, W., K. Flegal, et al. (2008). "Heart disease and stroke statistics--2008 update: a report from the American Heart Association Statistics Committee and Stroke Statistics Subcommittee." Circulation 117(4): e25-146.

Sabatine, M. S., C. P. Cannon, et al. (2005). "Addition of clopidogrel to aspirin and fibrinolytic therapy for myocardial infarction with ST-segment elevation." N Engl J Med 352(12): 1179-89.

Sacks, F. M., M. A. Pfeffer, et al. (1996). "The effect of pravastatin on coronary events after myocardial infarction in patients with average cholesterol levels. Cholesterol and Recurrent Events Trial investigators." N Engl J Med 335(14): 1001-9.

Serebruany, V. L., A. I. Malinin, et al. (2004). "Risk of bleeding complications with antiplatelet agents: meta-analysis of 338,191 patients enrolled in 50 randomized controlled trials." Am J Hematol 75(1): 40-7.

Seshadri, N., P. L. Whitlow, et al. (2002). "Emergency coronary artery bypass surgery in the contemporary percutaneous coronary intervention era." Circulation 106(18): 2346-50.

Smith, S. C., Jr., J. Allen, et al. (2006). "AHA/ ACC guidelines for secondary prevention for patients with coronary and other atherosclerotic vascular disease: 2006 update: endorsed by the National Heart, Lung, and Blood Institute." Circulation 113(19): 2363-72.

Wallentin, L., R. C. Becker, et al. (2009). "Ticagrelor versus clopidogrel in patients with acute coronary syndromes." N Engl J Med 361(11): 1045-57.

Wiviott, S. D., E. Braunwald, et al. (2007). "Prasugrel versus clopidogrel in patients with acute coronary syndromes." N Engl J Med 357(20): 2001-15.

Yende, S. and R. G. Wunderink (2001). "Effect of clopidogrel on bleeding after coronary artery bypass surgery." Crit Care Med 29(12): 2271-5.

Yusuf, S., F. Zhao, et al. (2001). "Effects of clopidogrel in addition to aspirin in patients with acute coronary syndromes without ST-segment elevation." N Engl J Med 345(7): 494502. 


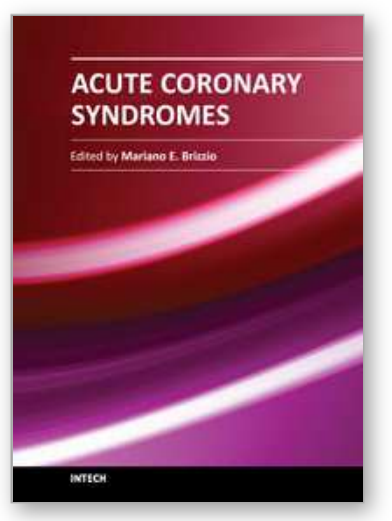

\author{
Acute Coronary Syndromes \\ Edited by Dr. Mariano Brizzio
}

ISBN 978-953-307-827-4

Hard cover, 214 pages

Publisher InTech

Published online 24, February, 2012

Published in print edition February, 2012

This book has been written with the intention of providing an up-to-the minute review of acute coronary syndromes. Atherosclerotic coronary disease is still a leading cause of death within developed countries and not surprisingly, is significantly rising in others. Over the past decade the treatment of these syndromes has changed dramatically. The introduction of novel therapies has impacted the outcomes and surviving rates in such a way that the medical community need to be up to date almost on a "daily bases". It is hoped that this book will provide a timely update on acute coronary syndromes and prove to be an invaluable resource for practitioners seeking new and innovative ways to deliver the best possible care to their patients.

\title{
How to reference
}

In order to correctly reference this scholarly work, feel free to copy and paste the following:

Stephen J. Huddleston and Gregory Trachiotis (2012). Coronary Bypass Grafting in Acute Coronary Syndrome: Operative Approaches and Secondary Prevention, Acute Coronary Syndromes, Dr. Mariano Brizzio (Ed.), ISBN: 978-953-307-827-4, InTech, Available from: http://www.intechopen.com/books/acute-coronarysyndromes/coronary-bypass-grafting-in-acute-coronary-syndrome-operative-approaches-and-secondaryprevention

\section{INTECH}

open science | open minds

\section{InTech Europe}

University Campus STeP Ri

Slavka Krautzeka 83/A

51000 Rijeka, Croatia

Phone: +385 (51) 770447

Fax: +385 (51) 686166

www.intechopen.com

\section{InTech China}

Unit 405, Office Block, Hotel Equatorial Shanghai

No.65, Yan An Road (West), Shanghai, 200040, China

中国上海市延安西路65号上海国际贵都大饭店办公楼 405 单元

Phone: +86-21-62489820

Fax: +86-21-62489821 
(C) 2012 The Author(s). Licensee IntechOpen. This is an open access article distributed under the terms of the Creative Commons Attribution 3.0 License, which permits unrestricted use, distribution, and reproduction in any medium, provided the original work is properly cited. 